\title{
Solution and Stability of a General Mixed Type Cubic and Quartic Functional Equation
}

\author{
Xiaopeng Zhao, ${ }^{1,2}$ Xiuzhong Yang, ${ }^{3}$ and Chin-Tzong Pang ${ }^{4}$ \\ ${ }^{1}$ Department of Mathematics, Zhejiang University, Hangzhou 310027, China \\ ${ }^{2}$ Department of Applied Mathematics, National Sun Yat-sen University, Kaohsiung 80424, Taiwan \\ ${ }^{3}$ College of Mathematics and Information Science, Hebei Normal University, \\ and Hebei Key Laboratory of Computational Mathematics and Applications, Shijiazhuang 050024, China \\ ${ }^{4}$ Department of Information Management, Yuan Ze University, Chung-Li 32003, Taiwan
}

Correspondence should be addressed to Chin-Tzong Pang; imctpang@saturn.yzu.edu.tw

Received 23 August 2013; Accepted 5 September 2013

Academic Editor: Jinlu Li

Copyright (C) 2013 Xiaopeng Zhao et al. This is an open access article distributed under the Creative Commons Attribution License, which permits unrestricted use, distribution, and reproduction in any medium, provided the original work is properly cited.

We consider the following mixed type cubic and quartic functional equation $\lambda[f(x+\lambda y)+f(x-\lambda y)]=\lambda^{3}[f(x+y)+f(x-y)]-$ $2 \lambda^{3}(\lambda+1) f(y)-2 \lambda\left(\lambda^{2}-1\right) f(x)+2(\lambda+1) f(\lambda y)$, where $\lambda$ is a fixed integer. We establish the general solution of the functional equation when the integer $\lambda \neq 0, \pm 1$, and then, by using the fixed point alternative, we investigate the generalized Hyers-UlamRassias stability for this functional equation when the integer $\lambda \geq 2$.

\section{Introduction}

In 1940, Ulam [1] asked the fundamental question for the stability for the group homomorphisms.

Let $\left(G_{1}, *\right)$ be a group, and let $\left(G_{2}, \diamond, d\right)$ be a metric group with the metric $d(\cdot, \cdot)$. Given $\varepsilon>0$, does there exist a $\delta(\varepsilon)>0$ such that if a mapping $h: G_{1} \rightarrow G_{2}$ satisfies the inequality

$$
d(h(x * y), h(x) \diamond h(y))<\delta
$$

for all $x, y \in G_{1}$, then there is a homomorphism $H: G_{1} \rightarrow$ $G_{2}$ with

$$
d(h(x), H(x))<\varepsilon
$$

for all $x \in G_{1}$ ?

In other words, under what conditions, does there exist a homomorphism near an approximately homomorphism? In the next year, Hyers [2] gave the first affirmative answer to the question of Ulam for Cauchy equation in the Banach spaces. Then, Rassias [3] generalized Hyers' result by considering an unbounded Cauchy difference, and this stability phenomenon is known as generalized Hyers-Ulam-Rassias stability or Hyers-Ulam-Rassias stability. During the last three decades, the stability problem for several functional equations has been extensively investigated by many mathematicians; see, for example, [4-9] and the references therein. We also refer the readers to the books [10-13].

In [14], Jun and Kim introduced the following functional equation

$$
f(2 x+y)+f(2 x-y)=2 f(x+y)+2 f(x-y)+12 f(x) .
$$

It is easy to see that the function $f(x)=x^{3}$ satisfies the functional equation (3). Thus, it is natural that (3) is called a cubic functional equation and every solution of the cubic functional equation is said to be a cubic function. In [14], Jun and Kim established the general solution and the generalized Hyers-Ulam-Rassias stability for (3). They proved that a function $f: X \rightarrow Y$ between real vector spaces is a solution of the functional equation (3) if and only if there exists a function $G: X \times X \times X \rightarrow Y$ such that $f(x)=G(x, x, x)$ for all $x \in X$ and $G$ is symmetric for each fixed one variable and is additive for fixed two variables.

In [15], Lee et al. considered the following quartic functional equation

$$
\begin{aligned}
& f(2 x+y)+f(2 x-y) \\
& \quad=4[f(x+y)+f(x-y)]+24 f(x)-6 f(y) .
\end{aligned}
$$


Since the function $f(x)=x^{4}$ satisfies the functional equation (4), the functional equation (4) is called a quartic functional equation and every solution of the quartic functional equation is said to be a quartic function. In [15], the authors solved the functional equation (4) and proved the stability for it. Actually, they obtained that a function $f: X \rightarrow Y$ between real vector spaces satisfies the functional equation (4) if and only if there exists a symmetric biquadratic function $B: X \times X \rightarrow Y$ such that $f(x)=B(x, x)$ for all $x \in X$. A function $f: X \rightarrow Y$ between real vector spaces is said to be quadratic if it satisfies the following functional equation

$$
f(x+y)+f(x-y)=2 f(x)+2 f(y)
$$

for all $x, y \in X$, and a function $B: X \times X \rightarrow Y$ is said to be bi-quadratic if $B$ is quadratic for each fixed one variable (see [8]). The following mixed type cubic and quartic functional equation was introduced by Eshaghi Gordji et al. [16]

$$
\begin{aligned}
f(x+2 y)+f(x-2 y) & \\
= & 4[f(x+y)+f(x-y)] \\
& -24 f(y)-6 f(x)+3 f(2 y) .
\end{aligned}
$$

It can be verified that the function $f(x)=x^{3}+x^{4}$ satisfies the functional equation (6). In [16], the authors obtained the general solution and the generalized Hyers-Ulam-Rassias stability of (6) in quasi-Banach space. The literature on the stability of the mixed type functional equations is very rich; see [17-22].

In the present paper, we extend (6) and consider the following functional equation

$$
\begin{aligned}
\lambda[f(x+\lambda y)+f(x-\lambda y)] & \\
= & \lambda^{3}[f(x+y)+f(x-y)]-2 \lambda^{3}(\lambda+1) f(y) \\
& -2 \lambda\left(\lambda^{2}-1\right) f(x)+2(\lambda+1) f(\lambda y),
\end{aligned}
$$

where $\lambda$ is a fixed integer. One can see that the functional equation (6) is a special case of (7) when we take the integer $\lambda=2$.

In 2003, Radu [23] noticed that the fixed point theorem, which was established by Diaz and Margolis [24], plays an important part in solving the stability problem of functional equations. Subsequently, this method has been successfully used by many mathematicians to investigate the stability of several functional equations; see, for example, $[21,25,26]$ and the references therein.

In this paper, we first establish the general solution of functional equation (7) when the integer $\lambda \neq 0, \pm 1$ and $f$ is a mapping between vector spaces. Then, by using the fixed point method, we prove the generalized Hyers-Ulam-Rassias stability of the functional equation (7) when the integer $\lambda \geq 2$ and $f$ is a mapping from the normed space to the Banach space.

\section{Solution of the Functional Equation (7)}

Recall form $[14,15]$ that every solution of the cubic functional equation (3) and the quartic functional equation (4) is said to be a cubic function and a quartic function, respectively. In this section, we investigate the general solution of the mixed type cubic and quartic functional equation (7). Throughout this section, let $X$ and $Y$ be two real vector spaces, and we always assume that the integer $\lambda$ in the functional equation (7) is different from $0,-1$, and 1 . Before proving our main theorem, we first give the following two lemmas.

Lemma 1. If an odd mapping $f: X \rightarrow Y$ satisfies (7), then $f$ is cubic.

Proof. Note that, in view of the oddness of $f$, we have $f(0)=$ 0 and $f(-x)=-f(x)$ for all $x \in X$. Letting $x=0$ in (7), we get

$$
f(\lambda y)=\lambda^{3} f(y)
$$

for all $y \in X$. Applying (8) to (7), we obtain

$$
\begin{aligned}
f(x+ & +\lambda y)+f(x-\lambda y) \\
& =\lambda^{2}[f(x+y)+f(x-y)]-2\left(\lambda^{2}-1\right) f(x)
\end{aligned}
$$

for all $x, y \in X$. Replacing $y$ by $x+y$ in (9), we get

$$
\begin{aligned}
f((\lambda & +1) x+\lambda y)-f((\lambda-1) x+\lambda y) \\
& =\lambda^{2}[f(2 x+y)-f(y)]-2\left(\lambda^{2}-1\right) f(x)
\end{aligned}
$$

for all $x, y \in X$. Now, if we replace $x$ by $\lambda x+y$ in (9) and use (8), we see that

$$
\begin{aligned}
f(\lambda x & +(\lambda+1) y)+f(\lambda x-(\lambda-1) y) \\
& =\lambda^{2}\left[f(\lambda x+2 y)+\lambda^{3} f(x)\right]-2\left(\lambda^{2}-1\right) f(\lambda x+y)
\end{aligned}
$$

for all $x, y \in X$. Interchanging $x$ with $y$ in (11) and using the oddness of $f$, we get the relation

$$
\begin{aligned}
f((\lambda & +1) x+\lambda y)-f((\lambda-1) x-\lambda y) \\
& =\lambda^{2}\left[f(2 x+\lambda y)+\lambda^{3} f(y)\right]-2\left(\lambda^{2}-1\right) f(x+\lambda y)
\end{aligned}
$$

for all $x, y \in X$. Then, subtracting (12) from (10), one has

$$
\begin{aligned}
f((\lambda-1) x-\lambda y)-f((\lambda-1) x+\lambda y) \\
=\lambda^{2}[f(2 x+y)-f(2 x+\lambda y)] \\
\quad-\left(\lambda^{2}+\lambda^{5}\right) f(y)+2\left(\lambda^{2}-1\right)(f(x+\lambda y)-f(x))
\end{aligned}
$$

for all $x, y \in X$. Now, replacing $y$ by $-y$ in (13) and noting that $f$ is odd, we have

$$
\begin{aligned}
f((\lambda-1) x+\lambda y)-f((\lambda-1) x-\lambda y) & \\
= & \lambda^{2}[f(2 x-y)-f(2 x-\lambda y)]+\left(\lambda^{2}+\lambda^{5}\right) f(y) \\
& +2\left(\lambda^{2}-1\right)(f(x-\lambda y)-f(x))
\end{aligned}
$$


for all $x, y \in X$. Adding (13) to (14) gives

$$
\begin{aligned}
\lambda^{2}[f(2 x+y)+f(2 x-y)] \\
\quad+2\left(\lambda^{2}-1\right)[f(x+\lambda y)+f(x-\lambda y)] \\
\quad=\lambda^{2}[f(2 x+\lambda y)+f(2 x-\lambda y)]+4\left(\lambda^{2}-1\right) f(x)
\end{aligned}
$$

for all $x, y \in X$. Replacing $x$ by $2 x$ in (9) and using (8), we get

$$
\begin{aligned}
f(2 x & +\lambda y)+f(2 x-\lambda y) \\
& =\lambda^{2}[f(2 x+y)+f(2 x-y)]-16\left(\lambda^{2}-1\right) f(x) .
\end{aligned}
$$

Applying (9) and (16) to (15) yields that

$$
\begin{aligned}
f(2 x & +y)+f(2 x-y) \\
& =2[f(x+y)+f(x-y)]+12 f(x)
\end{aligned}
$$

for all $x, y \in X$. Thus, the mapping $f: X \rightarrow Y$ is cubic. This completes the proof.

Lemma 2. If an even mapping $f: X \rightarrow Y$ satisfies (7) for all $x, y \in X$, then $f$ is quartic.

Proof. In view of the evenness of $f$, we have $f(-x)=f(x)$ for all $x \in X$. Putting $x=y=0$ in (7), we get $f(0)=0$. Then, let $x=0$ in (7), we obtain

$$
f(\lambda y)=\lambda^{4} f(y)
$$

for all $y \in X$. Combing (7) and (18) implies the following equation

$$
\begin{aligned}
f(x+ & \lambda y)+f(x-\lambda y) \\
= & \lambda^{2}[f(x+y)+f(x-y)] \\
& -2\left(\lambda^{2}-1\right) f(x)+2 \lambda^{2}\left(\lambda^{2}-1\right) f(y)
\end{aligned}
$$

for all $x, y \in X$. Replacing $y$ by $x+y$ in (19) and note that $f$ is even, we get

$$
\begin{aligned}
& f((\lambda+1) x+\lambda y)+f((\lambda-1) x+\lambda y) \\
&= \lambda^{2}[f(2 x+y)+f(y)]-2\left(\lambda^{2}-1\right) f(x) \\
&+2 \lambda^{2}\left(\lambda^{2}-1\right) f(x+y)
\end{aligned}
$$

for all $x, y \in X$. Replacing $x$ by $\lambda x+y$ in (19) and using (18), we get

$$
\begin{aligned}
f(\lambda x+ & (\lambda+1) y)+f(\lambda x-(\lambda-1) y) \\
= & \lambda^{2}\left[f(\lambda x+2 y)+\lambda^{4} f(x)\right] \\
& -2\left(\lambda^{2}-1\right) f(\lambda x+y)+2 \lambda^{2}\left(\lambda^{2}-1\right) f(y)
\end{aligned}
$$

for all $x, y \in X$. Interchanging the roles of $x$ and $y$ in (21), we obtain

$$
\begin{aligned}
f((\lambda+1) x+\lambda y)+f((\lambda-1) x-\lambda y) \\
=\lambda^{2}\left[f(2 x+\lambda y)+\lambda^{4} f(y)\right] \\
\quad-2\left(\lambda^{2}-1\right) f(x+\lambda y)+2 \lambda^{2}\left(\lambda^{2}-1\right) f(x)
\end{aligned}
$$

for all $x, y \in X$. If we subtract (22) from (20), we obtain

$$
\begin{aligned}
& f((\lambda-1) x+\lambda y)-f((\lambda-1) x-\lambda y) \\
&= \lambda^{2}[f(2 x+y)-f(2 x+\lambda y)] \\
&+2\left(\lambda^{2}-1\right) f(x+\lambda y)+\left(\lambda^{2}-\lambda^{6}\right) f(y) \\
&+2 \lambda^{2}\left(\lambda^{2}-1\right) f(x+y)-2\left(\lambda^{2}+1\right)\left(\lambda^{2}-1\right) f(x)
\end{aligned}
$$

for all $x, y \in X$. Replacing $y$ by $-y$ in (23), we get

$$
\begin{aligned}
& f((\lambda-1) x-\lambda y)-f((\lambda-1) x+\lambda y) \\
&= \lambda^{2}[f(2 x-y)-f(2 x-\lambda y)] \\
&+2\left(\lambda^{2}-1\right) f(x-\lambda y)+\left(\lambda^{2}-\lambda^{6}\right) f(y) \\
&+2 \lambda^{2}\left(\lambda^{2}-1\right) f(x-y)-2\left(\lambda^{2}+1\right)\left(\lambda^{2}-1\right) f(x)
\end{aligned}
$$

for all $x, y \in X$. If we add (23) to (24), we have

$$
\begin{aligned}
\lambda^{2}[f(2 x & +y)+f(2 x-y)] \\
& -\lambda^{2}[f(2 x+\lambda y)+f(2 x-\lambda y)] \\
& +2\left(\lambda^{2}-1\right)[f(x+\lambda y)+f(x-\lambda y)] \\
& +2 \lambda^{2}\left(\lambda^{2}-1\right)[f(x+y)+f(x-y)] \\
= & 4\left(\lambda^{2}+1\right)\left(\lambda^{2}-1\right) f(x)+2\left(\lambda^{6}-\lambda^{2}\right) f(y)
\end{aligned}
$$

for all $x, y \in X$. Replacing $x$ by $2 x$ in (19) and using (18), we get

$$
\begin{aligned}
f(2 x+ & \lambda y)+f(2 x-\lambda y) \\
= & \lambda^{2}[f(2 x+y)+f(2 x-y)] \\
& -32\left(\lambda^{2}-1\right) f(x)+2 \lambda^{2}\left(\lambda^{2}-1\right) f(y)
\end{aligned}
$$

for all $x, y \in X$. Applying (19) and (26) to (25), we obtain that

$$
\begin{aligned}
f(2 x & +y)+f(2 x-y) \\
& =4[f(x+y)+f(x-y)]+24 f(x)-6 f(y)
\end{aligned}
$$

for all $x, y \in X$. Therefore, the mapping $f: X \rightarrow Y$ is quartic and the proof is complete.

Now, we are ready to find out the general solution of (7). 
Theorem 3. A mapping $f: X \rightarrow Y$ satisfies (7) for all $x, y \in$ $X$ if and only if there exist a symmetric multiadditive mapping $G: X \times X \times X \rightarrow Y$ and a symmetric bi-quadratic mapping $B: X \times X \rightarrow Y$ such that $f(x)=G(x, x, x)+B(x, x)$ for all $x \in X$.

Proof. First, we assume that there exist a symmetric multiadditive mapping $G: X \times X \times X \rightarrow Y$ and a symmetric bi-quadratic mapping $B: X \times X \rightarrow Y$ such that $f(x)=$ $G(x, x, x)+B(x, x)$ for all $x \in X$. We need to show that the function $f$ satisfies (7). By a simple computation, one can obtain that the function $x \mapsto G(x, x, x)$ satisfies (7). Thus, to show that the function $f$ satisfies (7), we only need to show that the function $x \mapsto B(x, x)$ also satisfies (7); namely,

$$
\begin{aligned}
\lambda[B(x & +\lambda y, x+\lambda y)+B(x-\lambda y, x-\lambda y)] \\
= & \lambda^{3}[B(x+y, x+y)+B(x-y, x-y)] \\
& -2 \lambda^{3}(\lambda+1) B(y, y) \\
& -2 \lambda\left(\lambda^{2}-1\right) B(x, x)+2(\lambda+1) B(\lambda y, \lambda y)
\end{aligned}
$$

for all $x, y \in X$. Since $B: X \times X \rightarrow Y$ is a bi-quadratic mapping, it can be verified that

$$
B(\lambda x, \lambda y)=\lambda^{4} B(x, y)
$$

for all integer $\lambda$ and all $x, y \in X$. Then, (28) becomes

$$
\begin{aligned}
B(x+ & \lambda y, x+\lambda y)+B(x-\lambda y, x-\lambda y) \\
= & \lambda^{2}[B(x+y, x+y)+B(x-y, x-y)] \\
& \quad-2\left(\lambda^{2}-1\right) B(x, x)+2 \lambda^{2}\left(\lambda^{2}-1\right) B(y, y) .
\end{aligned}
$$

To establish (28), it suffices to show (30). Note that if (30) holds for some integer $\lambda$, then so does $-\lambda$. Thus, in the following, we will show that (30) holds for all positive integers $\lambda$ with $\lambda \geq 2$ and all $x, y \in X$. To do this, we use induction on $\lambda$. Fix any $x, y \in X$. In the case when $\lambda=2$, we have

$$
\begin{aligned}
& B(x+2 y, x+2 y)+B(x-2 y, x-2 y) \\
&= {[B(x+y+y, x+2 y)+B(x+y-y, x+2 y)] } \\
&+[B(x-y+y, x-2 y)+B(x-y-y, x-2 y)] \\
&-[B(x, x+2 y)+B(x, x-2 y)] \\
&= 2[B(x+y, x+2 y)+B(y, x+2 y)] \\
&+2[B(x-y, x-2 y)+B(y, x-2 y)] \\
&-[2 B(x, x)+2 B(x, 2 y)] \\
&= 2[B(x+y, x+y+y)+B(x+y, x+y-y)] \\
&+2[B(x-y, x-y-y)+B(x-y, x-y+y)] \\
&-2[B(x+y, x)+B(x-y, x)] \\
&+2[B(y, x+2 y)+B(y, x-2 y)] \\
&-[2 B(x, x)+8 B(x, y)]
\end{aligned}
$$

$$
\begin{aligned}
= & 4[B(x+y, x+y)+B(x+y, y)] \\
& +4[B(x-y, x-y)+B(x-y, y)] \\
& -4[B(x, x)+B(y, x)]+4[B(y, x)+B(y, 2 y)] \\
& -[2 B(x, x)+8 B(x, y)] \\
= & 4[B(x+y, x+y)+B(x-y, x-y)] \\
& +4[B(x+y, y)+B(x-y, y)] \\
& -6 B(x, x)-8 B(x, y)+16 B(y, y) \\
= & 4[B(x+y, x+y)+B(x-y, x-y)] \\
& +8[B(x, y)+B(y, y)] \\
& -6 B(x, x)-8 B(x, y)+16 B(y, y) \\
= & 4[B(x+y, x+y)+B(x-y, x-y)] \\
& -6 B(x, x)+24 B(y, y) .
\end{aligned}
$$

So (30) is true for $\lambda=2$. Here, we have used the bi-quadratic property of the function $B$ and (29). Now, assume that (30) is true for all positive integers that are less than or equal to some integer $\lambda(>2)$. Then,

$$
\begin{aligned}
B(x+ & (\lambda+1) y, x+(\lambda+1) y) \\
+ & B(x-(\lambda+1) y, x-(\lambda+1) y) \\
= & {[B(x+y+\lambda y, x+y+\lambda y)} \\
+ & B(x+y-\lambda y, x+y-\lambda y)] \\
+ & {[B(x-y+\lambda y, x-y+\lambda y)} \\
& +B(x-y-\lambda y, x-y-\lambda y)] \\
- & {[B(x+(\lambda-1) y, x+(\lambda-1) y)} \\
& +B(x-(\lambda-1) y, x-(\lambda-1) y)] \\
= & \lambda^{2}[B(x+2 y, x+2 y)+B(x, x)] \\
- & 2\left(\lambda^{2}-1\right) B(x+y, x+y)+2 \lambda^{2}\left(\lambda^{2}-1\right) B(y, y) \\
+ & \lambda^{2}[B(x, x)+B(x-2 y, x-2 y)] \\
- & +2\left(n^{2}-1\right) B(x-y, x-y)+2 \lambda^{2}\left(\lambda^{2}-1\right) B(y, y) \\
- & \left\{(\lambda-1)^{2}[B(x+y, x+y)+B(x-y, x-y)]\right. \\
& -2\left((\lambda-1)^{2}-1\right) B(x, x) \\
& \\
& \left.\left.+(\lambda-1)^{2}-1\right) B(y, y)\right\} .
\end{aligned}
$$


Applying (31) to (32), one can obtain that

$$
\begin{aligned}
B(x+ & (\lambda+1) y, x+(\lambda+1) y) \\
+ & B(x-(\lambda+1) y, x-(\lambda+1) y) \\
= & (\lambda+1)^{2}[B(x+y, x+y)+B(x-y, x-y)] \\
& -2\left((\lambda+1)^{2}-1\right) B(x, x) \\
& +2(\lambda+1)^{2}\left((\lambda+1)^{2}-1\right) B(y, y) .
\end{aligned}
$$

This means that (30) is true for $\lambda+1$, and we have showed that the function $x \mapsto B(x, x)$ satisfies (7). Therefore, the mapping $f: X \rightarrow Y$ satisfies (7).

Conversely, we decompose $f$ into the odd part and the even part by putting

$$
f_{o}(x)=\frac{f(x)-f(-x)}{2}, \quad f_{e}(x)=\frac{f(x)+f(-x)}{2}
$$

for all $x \in X$. Then, $f(x)=f_{o}(x)+f_{e}(x)$ for all $x \in X$. It is easy to show that the mappings $f_{o}$ and $f_{e}$ satisfy (7). Hence, it follows from Lemmas 1 and 2 that the function $f_{o}$ is cubic and $f_{e}$ is quartic, respectively. Therefore, there exist a symmetric multi-additive mapping $G: X \times X \times X \rightarrow Y$ such that $f_{o}(x)=G(x, x, x)$ for all $x \in X$ (see [14, Theorem 2.1]) and a symmetric bi-quadratic mapping $B: X \times X \rightarrow Y$ such that $f_{e}(x)=B(x, x)$ for all $x \in X$ (see [15, Theorem 2.1]). Hence, we get $f(x)=G(x, x, x)+B(x, x)$ for all $x \in X$. The proof is complete.

\section{Generalized Hyers-Ulam-Rassias Stability of the Functional Equation (7)}

In this section, we will investigate the stability of the functional equation (7) by using the fixed point alternative. Throughout this section, let $X$ be a real normed space and $Y$ be a real Banach space, and we always assume that the integer $\lambda$ used in the section is greater than or equal to 2 . For convenience, we use the following abbreviation for a given function $f: X \rightarrow$ $Y$ :

$$
\begin{aligned}
D f(x, y):= & \lambda[f(x+\lambda y)+f(x-\lambda y)] \\
& -\lambda^{3}[f(x+y)+f(x-y)] \\
& +2 \lambda^{3}(\lambda+1) f(y)+2 \lambda\left(\lambda^{2}-1\right) f(x) \\
& -2(\lambda+1) f(\lambda y)
\end{aligned}
$$

for all $x, y \in X$.

Let us recall the following result by Diaz and Margolis.

Proposition 4 (see [24]). Let $(E, d)$ be a complete generalized metric space (i.e., one for which d may assume infinite value), and let $J: E \rightarrow E$ be a strictly contractive mapping with Lipschitz constant $L<1$; that is,

$$
d(J x, J y) \leq L d(x, y) \quad \forall x, y \in X
$$

Then, for each fixed element $x_{0} \in E$, either

$$
d\left(J^{n} x_{0}, J^{n+1} x_{0}\right)=+\infty
$$

for all nonnegative integers $n$ or there exists a non-negative integer $n_{0}$ such that

(a) $d\left(J^{n} x_{0}, J^{n+1} x_{0}\right)<+\infty$ for all $n \geq n_{0}$;

(b) the sequence $\left\{J^{n} x_{0}\right\}$ converges to a fixed point $x^{*}$ of $J$;

(c) $x^{*}$ is the unique fixed point of $J$ in the set $\mathscr{F}:=\{x \in E \mid$ $\left.d\left(J^{n_{0}} x_{0}, x\right)<+\infty\right\}$;

(d) $d\left(x, x^{*}\right) \leq(1 /(1-L)) d(x, J x)$ for all $x \in \mathscr{F}$.

Lemma 5. Let $f: X \rightarrow Y$ be an odd function for which there exists a function $\varphi: X \times X \rightarrow[0,+\infty)$ such that

$$
\|D f(x, y)\| \leq \varphi(x, y)
$$

for all $x, y \in X$. If there exists a constant $0<L<1$ such that

$$
\varphi(\lambda x, \lambda y) \leq \lambda^{3} L \varphi(x, y)
$$

for all $x, y \in X$, then there exists a unique cubic mapping $C$ : $X \rightarrow Y$ such that

$$
\|f(x)-C(x)\| \leq \frac{L}{1-L} \phi(x)
$$

for all $x \in X$, where $\phi(x):=(1 / 2(\lambda+1)) \varphi(0, x / \lambda)$.

Proof. It follows from (39) that

$$
\lim _{r \rightarrow \infty} \frac{\varphi\left(\lambda^{r} x, \lambda^{r} y\right)}{\lambda^{3 r}}=0
$$

for all $x, y \in X$. Letting $x=0$ in (38) and replacing $y$ by $x$, we have

$$
\left\|\lambda^{3} f(x)-f(\lambda x)\right\| \leq \phi(\lambda x)
$$

for all $x \in X$. By (39), we have $\phi(\lambda x) \leq \lambda^{3} L \phi(x)$ for all $x \in X$. This, together with (42), implies that

$$
\left\|f(x)-\frac{1}{\lambda^{3}} f(\lambda x)\right\| \leq L \phi(x)
$$

for all $x \in X$. Let $\Omega$ be the set of all odd mappings $g: X \rightarrow Y$. We introduce the generalized metric on $\Omega$ :

$$
\begin{aligned}
& d(g, h) \\
& \quad:=\inf \{K \in[0, \infty) \mid\|g(x)-h(x)\| \leq K \phi(x), x \in X\} .
\end{aligned}
$$

It is easy to show that $(\Omega, d)$ is complete. Now, we define a function $T: \Omega \rightarrow \Omega$ by

$$
\operatorname{Tg}(x):=\frac{1}{\lambda^{3}} g(\lambda x)
$$


for all $g \in \Omega$ and all $x \in X$. Note that, for all $g, h \in \Omega$,

$$
\begin{aligned}
& d(g, h) \leq K \\
& \quad \Longrightarrow\|g(x)-h(x)\| \leq K \phi(x), \quad x \in X \\
& \Longrightarrow\left\|\frac{1}{\lambda^{3}} g(\lambda x)-\frac{1}{\lambda^{3}} h(\lambda x)\right\| \leq \frac{1}{\lambda^{3}} K \phi(\lambda x), \quad x \in X \\
& \Longrightarrow\left\|\frac{1}{\lambda^{3}} g(\lambda x)-\frac{1}{\lambda^{3}} h(\lambda x)\right\| \leq L K \phi(x), \quad x \in X \\
& \Longrightarrow d(T g, T h) \leq L K .
\end{aligned}
$$

Hence, we obtain that

$$
d(T g, T h) \leq L d(g, h)
$$

for all $g, h \in \Omega$; that is, $T$ is a strictly contractive mapping of $\Omega$ with Lipschitz constant $L$. It follows from (43) that $d(T f, f) \leq$ $L$. Therefore, according to Proposition 4, the sequence $\left\{T^{r} f\right\}$ converges to a fixed point $C$ of $T$; that is,

$$
C: X \rightarrow Y, \quad C(x)=\lim _{r \rightarrow \infty}\left(T^{r} f\right)(x)=\lim _{r \rightarrow \infty} \frac{f\left(\lambda^{r} x\right)}{\lambda^{3 r}}
$$

and $C(\lambda x)=\lambda^{3} C(x)$ for all $x \in X$. Also $C$ is the unique fixed point of $T$ in the set $\Delta=\{g \in \Omega \mid d(f, g)<\infty\}$ and

$$
d(f, C) \leq \frac{1}{1-L} d(T f, f) \leq \frac{L}{1-L},
$$

which yields the inequality (40). It follows from the definition of $C,(38)$, and (41) that

$$
\|D C(x, y)\|=\lim _{r \rightarrow \infty} \frac{\left\|D f\left(\lambda^{r} x, \lambda^{r} y\right)\right\|}{\lambda^{3 r}} \leq \lim _{r \rightarrow \infty} \frac{\varphi\left(\lambda^{r} x, \lambda^{r} y\right)}{\lambda^{3 r}}=0
$$

for all $x, y \in X$; that is, the mapping $C: X \rightarrow Y$ satisfies (7). Since $f$ is odd, $C$ is odd. Therefore, Lemma 1 guarantees that $C$ is cubic. Finally, it remains to prove the uniqueness of $C$. Let $S: X \rightarrow Y$ be another cubic function satisfying (40). Since $d(f, S) \leq L /(1-L)$ and $S$ is cubic, we get $S \in \Delta$ and $T S(x)=\left(1 / \lambda^{3}\right) S(\lambda x)=S(x)$ for all $x \in X$; that is, $S$ is a fixed point of $T$. Since $C$ is the unique fixed point of $T$ in $\Delta$, it follows that $S=C$.

Lemma 6. Let $f: X \rightarrow Y$ be an odd function for which there exists a function $\varphi: X \times X \rightarrow[0,+\infty)$ such that

$$
\|D f(x, y)\| \leq \varphi(x, y)
$$

for all $x, y \in X$. If there exists a constant $0<L<1$ such that

$$
\lambda^{3} \varphi(x, y) \leq L \varphi(\lambda x, \lambda y)
$$

for all $x, y \in X$, then there exists a unique cubic mapping $C$ : $X \rightarrow Y$ such that

$$
\|f(x)-C(x)\| \leq \frac{1}{1-L} \psi(x)
$$

for all $x \in X$, where $\psi(x):=(1 / 2(\lambda+1)) \varphi(0, x / \lambda)$.
Proof. It follows from (52) that

$$
\lim _{r \rightarrow \infty} \lambda^{3 r} \varphi\left(\frac{x}{\lambda^{r}}, \frac{y}{\lambda^{r}}\right)=0
$$

for all $x, y \in X$. Letting $x=0$ in (51) and replacing $y$ by $x$, we have

$$
\left\|\lambda^{3} f\left(\frac{x}{\lambda}\right)-f(x)\right\| \leq \psi(x)
$$

for all $x \in X$. We introduce the same definitions for $\Omega$ and $d$ as in the proof of Lemma 5 (by replacing $\phi$ by $\psi$ ) such that $(\Omega, d$ ) becomes a generalized complete metric space. Let $T: \Omega \rightarrow \Omega$ be the mapping defined by

$$
\operatorname{Tg}(x):=\lambda^{3} g\left(\frac{x}{\lambda}\right)
$$

for all $g \in \Omega$ and all $x \in X$. One can show that $d(T g, T h) \leq$ $L d(g, h)$ for all $g, h \in \Omega$. It follows from (55) that $d(T f, f) \leq 1$. Due to Proposition 4, the sequence $\left\{T^{r} f\right\}$ converges to a fixed point $C$ of $T$; that is,

$$
C: X \rightarrow Y, \quad C(x)=\lim _{r \rightarrow \infty}\left(T^{r} f\right)(x)=\lim _{r \rightarrow \infty} \lambda^{3 r} f\left(\frac{x}{\lambda^{r}}\right)
$$

and $C(\lambda x)=\lambda^{3} C(x)$ for all $x \in X$. Also,

$$
d(f, C) \leq \frac{1}{1-L} d(T f, f) \leq \frac{1}{1-L}
$$

which yields the inequality (53). The rest of the proof is similar to the proof of Lemma 5, and we omit the details.

Similarly, we can prove the following two lemmas on even functions.

Lemma 7. Let $f: X \rightarrow Y$ be an even function with $f(0)=0$ for which there exists a function $\varphi: X \times X \rightarrow[0,+\infty)$ such that

$$
\|D f(x, y)\| \leq \varphi(x, y)
$$

for all $x, y \in X$. If there exists a constant $0<L<1$ such that

$$
\varphi(\lambda x, \lambda y) \leq \lambda^{4} L \varphi(x, y)
$$

for all $x, y \in X$, then there exists a unique quartic mapping $Q: X \rightarrow Y$ such that

$$
\|f(x)-Q(x)\| \leq \frac{L}{1-L} \phi(x)
$$

for all $x \in X$, where $\phi(x):=(1 / 2) \varphi(0, x / \lambda)$.

Lemma 8. Let $f: X \rightarrow Y$ be an even function with $f(0)=0$ for which there exists a function $\varphi: X \times X \rightarrow[0,+\infty)$ such that

$$
\|D f(x, y)\| \leq \varphi(x, y)
$$


for all $x, y \in X$. If there exists a constant $0<L<1$ such that

$$
\lambda^{4} \varphi(x, y) \leq L \varphi(\lambda x, \lambda y)
$$

for all $x, y \in X$, then there exists a unique quartic mapping $Q: X \rightarrow Y$ such that

$$
\|f(x)-Q(x)\| \leq \frac{1}{1-L} \psi(x)
$$

for all $x \in X$, where $\psi(x):=(1 / 2) \varphi(0, x / \lambda)$.

Now, we are ready to give our main theorems in this section.

Theorem 9. Let $f: X \rightarrow Y$ be a function with $f(0)=0$ for which there exists a function $\varphi: X \times X \rightarrow[0,+\infty)$ such that

$$
\|D f(x, y)\| \leq \varphi(x, y)
$$

for all $x, y \in X$. If there exists a constant $0<L<1$ such that

$$
\varphi(\lambda x, \lambda y) \leq \lambda^{3} L \varphi(x, y)
$$

for all $x, y \in X$, then there exist a unique cubic mapping $C$ : $X \rightarrow Y$ and $a$ unique quartic mapping $Q: X \rightarrow Y$ such that

$$
\|f(x)-C(x)-Q(x)\| \leq \frac{L}{1-L}\left[\phi_{o}(x)+\phi_{e}(x)\right]
$$

for all $x \in X$, where

$$
\begin{gathered}
\phi_{o}(x):=\frac{1}{2(\lambda+1)} \Phi\left(0, \frac{x}{\lambda}\right), \quad \phi_{e}(x):=\frac{1}{2} \Phi\left(0, \frac{x}{\lambda}\right), \\
\Phi(x, y):=\frac{1}{2}[\varphi(x, y)+\varphi(-x,-y)] .
\end{gathered}
$$

Proof. Let $f_{o}$ and $f_{e}$ denote the odd and the even part of $f$, respectively. Then, it can be verified from (65) that

$$
\left\|D f_{o}(x, y)\right\| \leq \Phi(x, y), \quad\left\|D f_{e}(x, y)\right\| \leq \Phi(x, y)
$$

for all $x, y \in X$. Moreover, by (66), it is easy to compute that

$$
\Phi(\lambda x, \lambda y) \leq \lambda^{3} L \Phi(x, y)
$$

for all $x, y \in X$. Thus, by applying Lemmas 5 and 7, one can obtain that there exist a unique cubic mapping $C: X \rightarrow Y$ and a unique quartic mapping $Q: X \rightarrow Y$ such that

$$
\begin{gathered}
\left\|f_{o}(x)-C(x)\right\| \leq \frac{L}{1-L} \phi_{o}(x), \\
\left\|f_{e}(x)-Q(x)\right\| \leq \frac{L}{1-L} \phi_{e}(x)
\end{gathered}
$$

for all $x \in X$, where $\phi_{o}(x):=(1 / 2(\lambda+1)) \Phi(0, x / \lambda)$ and $\phi_{e}(x):=(1 / 2) \Phi(0, x / \lambda)$. Moreover, combining (71) and (72) yields the inequality (67). The proof is complete.
Theorem 10. Let $f: X \rightarrow Y$ be a function with $f(0)=0$ for which there exists a function $\varphi: X \times X \rightarrow[0,+\infty)$ such that

$$
\|D f(x, y)\| \leq \varphi(x, y)
$$

for all $x, y \in X$. If there exists a constant $0<L<1$ such that

$$
\lambda^{4} \varphi(x, y) \leq L \varphi(\lambda x, \lambda y)
$$

for all $x, y \in X$, then there exist a unique cubic mapping $C$ : $X \rightarrow Y$ and $a$ unique quartic mapping $Q: X \rightarrow Y$ such that

$$
\|f(x)-C(x)-Q(x)\| \leq \frac{1}{1-L}\left[\psi_{o}(x)+\psi_{e}(x)\right]
$$

for all $x \in X$, where

$$
\begin{gathered}
\psi_{o}(x):=\frac{1}{2(\lambda+1)} \Psi\left(0, \frac{x}{\lambda}\right), \quad \psi_{e}(x):=\frac{1}{2} \Psi\left(0, \frac{x}{\lambda}\right), \\
\Psi(x, y):=\frac{1}{2}[\varphi(x, y)+\varphi(-x,-y)] .
\end{gathered}
$$

Proof. Similar to the proof of Theorem 9, the result follows from Lemmas 6 and 8.

\section{Conflict of Interests}

The authors declare that there is no conflict of interests regarding the publication of this paper.

\section{References}

[1] S. M. Ulam, Problems in Modern Mathematics, chapter 6, John Wiley \& Sons, New York, NY, USA, 1964.

[2] D. H. Hyers, "On the stability of the linear functional equation," Proceedings of the National Academy of Sciences of the United States of America, vol. 27, pp. 222-224, 1941.

[3] Th. M. Rassias, "On the stability of the linear mapping in Banach spaces," Proceedings of the American Mathematical Society, vol. 72, no. 2, pp. 297-300, 1978.

[4] S.-M. Jung, "Hyers-Ulam-Rassias stability of Jensen's equation and its application," Proceedings of the American Mathematical Society, vol. 126, no. 11, pp. 3137-3143, 1998.

[5] K.-W. Jun, H.-M. Kim, and I.-S. Chang, "On the Hyers-Ulam stability of an Euler-Lagrange type cubic functional equation," Journal of Computational Analysis and Applications, vol. 7, no. 1, pp. 21-33, 2005.

[6] K. W. Jun, S. B. Lee, and W. G. Park, "Solution and stability of a cubic functional equation," Acta Mathematica Sinica, vol. 26, no. 7, pp. 1255-1262, 2010.

[7] G. H. Kim, "A stability of the generalized sine functional equations," Journal of Mathematical Analysis and Applications, vol. 331, no. 2, pp. 886-894, 2007.

[8] W.-G. Park and J.-H. Bae, "On a bi-quadratic functional equation and its stability," Nonlinear Analysis. Theory, Methods \& Applications, vol. 62, no. 4, pp. 643-654, 2005.

[9] J. Sikorska, "Generalized stability of the Cauchy and the Jensen functional equations on spheres," Journal of Mathematical Analysis and Applications, vol. 345, no. 2, pp. 650-660, 2008. 
[10] J. Aczél and J. Dhombres, Functional Equations in Several Variables, vol. 31 of Encyclopedia of Mathematics and Its Applications, Cambridge University Press, 1989.

[11] S. Czerwik, Funcional Equations and Inequalities in Several Variables, World Scientific Publishing, River Edge, NJ, USA, 2002.

[12] D. H. Hyers, G. Isac, and Th. M. Rassias, Stability of Functional Equations in Several Variables, Birkhäuser, Boston, Mass, USA, 1998.

[13] Pl. Kannappan, Functional Equations and Inequalities with Applications, Springer Monographs in Mathematics, Springer, New York, NY, USA, 2009.

[14] K.-W. Jun and H.-M. Kim, "The generalized Hyers-UlamRassias stability of a cubic functional equation," Journal of Mathematical Analysis and Applications, vol. 274, no. 2, pp. 267-278, 2002.

[15] S. H. Lee, S. M. Im, and I. S. Hwang, "Quartic functional equations," Journal of Mathematical Analysis and Applications, vol. 307, no. 2, pp. 387-394, 2005.

[16] M. Eshaghi Gordji, S. Zolfaghari, J. M. Rassias, and M. B. Savadkouhi, "Solution and stability of a mixed type cubic and quartic functional equation in quasi-Banach spaces," Abstract and Applied Analysis, vol. 2009, Article ID 417473, 14 pages, 2009.

[17] M. E. Gordji, S. K. Gharetapeh, J. M. Rassias, and S. Zolfaghari, "Solution and stability of a mixed type additive, quadratic, and cubic functional equation," Advances in Difference Equations, vol. 2009, Article ID 826130, 17 pages, 2009.

[18] M. Eshaghi Gordji and H. Khodaei, "Solution and stability of generalized mixed type cubic, quadratic and additive functional equation in quasi-Banach spaces," Nonlinear Analysis. Theory, Methods \& Applications, vol. 71, no. 11, pp. 5629-5643, 2009.

[19] M. Eshaghi Gordji and M. B. Savadkouhi, "Stability of a mixed type cubic-quartic functional equation in non-Archimedean spaces," Applied Mathematics Letters, vol. 23, no. 10, pp. 11981202, 2010.

[20] F. Moradlou, H. Vaezi, and G. Z. Eskandani, "Hyers-UlamRassias stability of a quadratic and additive functional equation in quasi-Banach spaces," Mediterranean Journal of Mathematics, vol. 6, no. 2, pp. 233-248, 2009.

[21] A. Najati and Th. M. Rassias, "Stability of a mixed functional equation in several variables on Banach modules," Nonlinear Analysis. Theory, Methods \& Applications, vol. 72, no. 3-4, pp. 1755-1767, 2010.

[22] A. Najati and G. Z. Eskandani, "Stability of a mixed additive and cubic functional equation in quasi-Banach spaces," Journal of Mathematical Analysis and Applications, vol. 342, no. 2, pp.13181331, 2008.

[23] V. Radu, "The fixed point alternative and the stability of functional equations," Fixed Point Theory, vol. 4, no. 1, pp. 91-96, 2003.

[24] J. B. Diaz and B. Margolis, "A fixed point theorem of the alternative, for contractions on a generalized complete metric space," Bulletin of the American Mathematical Society, vol. 74, pp. 305309, 1968.

[25] L. Cădariu and V. Radu, "Fixed point methods for the generalized stability of functional equations in a single variable," Fixed Point Theory and Applications, vol. 2008, Article ID 749392, 15 pages, 2008.

[26] A. K. Mirmostafaee, "A fixed point approach to almost quartic mappings in quasi fuzzy normed spaces," Fuzzy Sets and Systems, vol. 160, no. 11, pp. 1653-1662, 2009. 


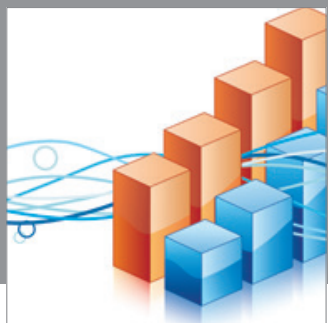

Advances in

Operations Research

mansans

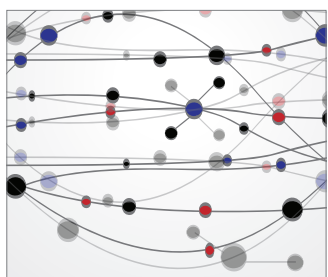

The Scientific World Journal
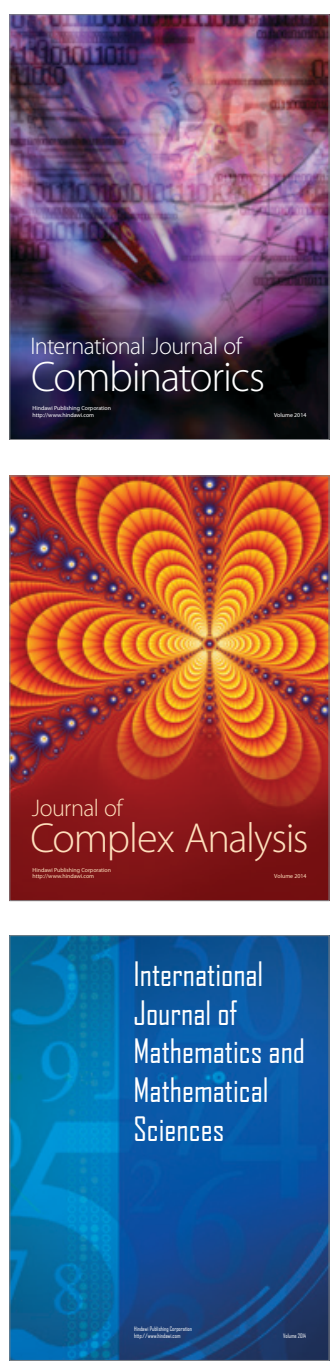
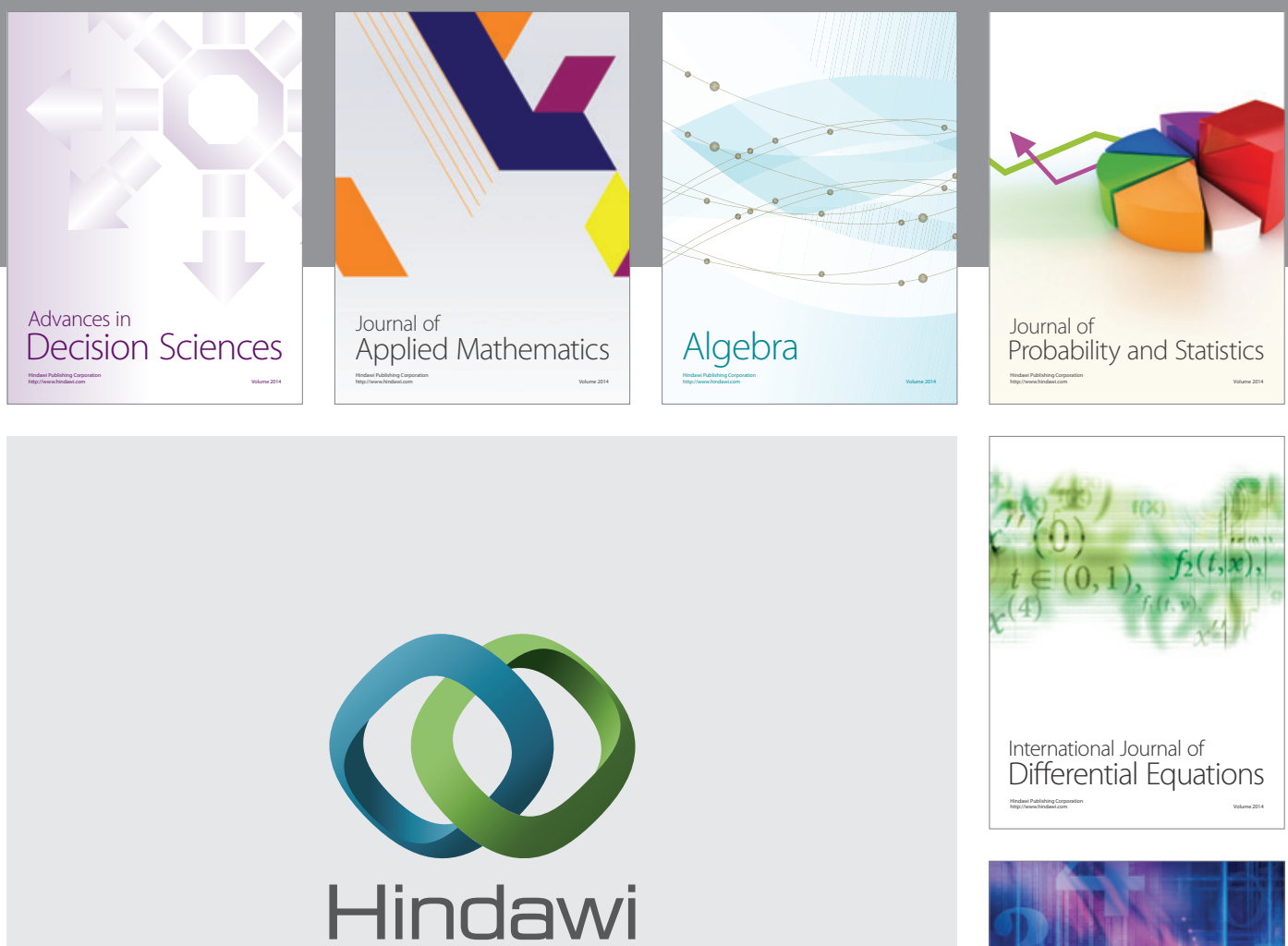

Submit your manuscripts at http://www.hindawi.com
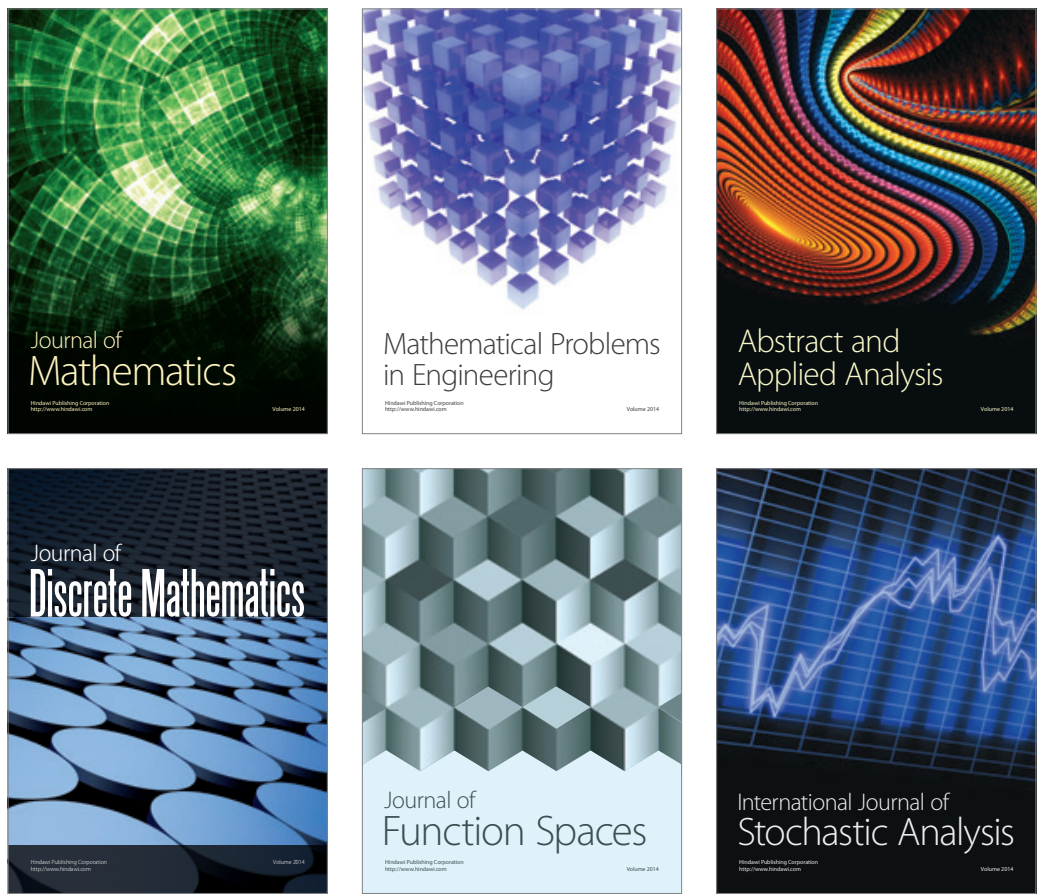

Journal of

Function Spaces

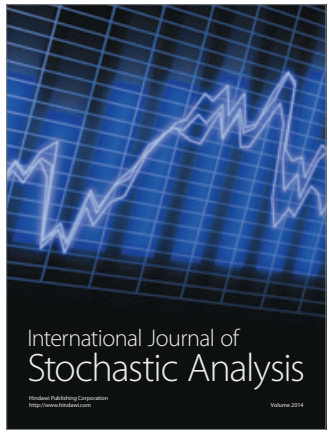

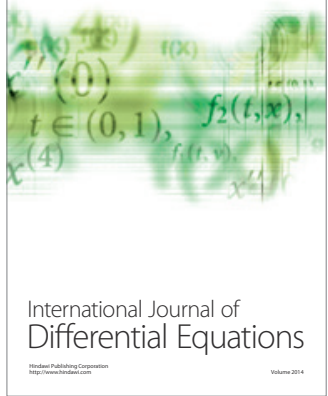
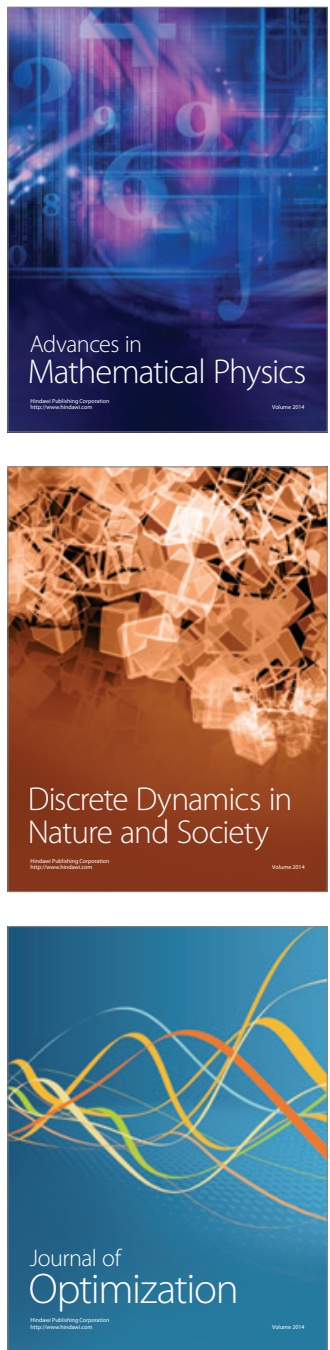